\title{
Detección de polyomavirus BK y JC en extractos leucocitarios de sangre periférica de pacientes con infección por VIH del área norte de Santiago
}

\author{
María José Martínez, Claudia Moreno, Jorge Levican, Mónica Peña, Aldo Gaggero y Jonás Chnaiderman
}

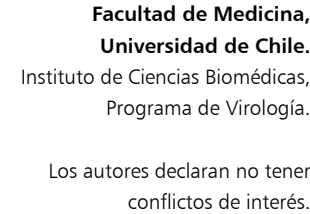

Fuente de financiamiento: Programa de Virología, I.C.B.M., Facultad de Medicina, Universidad de Chile.

Recibido: 23 de julio de 2015 Aceptado: 29 de enero de 2016

Correspondencia a: Jonás Chnaiderman F. jchnaiderman@med.uchile.cl

\section{BK and JC polyomavirus detection in leukocyte extracts of peripheral blood samples of HIV+ patients from the north area of Santiago}

Introduction: Polyomavirus $\mathrm{BK}(\mathrm{BKPyV})$ and $\mathrm{JC}(\mathrm{JCPyV})$ are persistent pathogens able to reactivate in immunocompromised patients, involving mostly urinary and central nervous system. There are no Chilean studies in HIV positive patients. Objective: To detect BKPyV and JCPyV in blood of Chilean HIV positive adult patients and to correlate these results with clinical-related variables. Materials and Methods: 96 stored blood samples from HIV patients belonging to the north area of Santiago were analyzed. Viral genomes of both viruses were detected by real-time PCR. For statistical analysis, chi-square (Pearson) and Mann-Whitney tests were used and p-values $<0.05$ were considered significant. Results: $33 \%$ of the samples were positive for BKPyV and a significant correlation was found between the presence of BKPyV genome and the absence of detectable HIV viral load. We demonstrated the need to consider more than one amplification target to detect the BKPyV genome. All the samples were negative for JCPyV genome. Discussion: BKPyV prevalence in Chilean HIV patients is higher than most of international studies. New studies regarding the interaction between both viruses are required. These patients should undergo periodic evaluations by urologist and nephrologist.

Key words: Polyomavirus, BK virus, JC virus, prevalence, HIV.

Palabras clave: Polyomavirus, virus BK, virus JC, prevalencia, VIH.

\section{Introducción}

$\checkmark$ 1 virus BK (BKPyV) fue descrito por primera vez el año $1971^{1}$ en muestras de orina de pacientes con trasplante renal que presentaron obstrucción ureteral.

Posteriormente, se describió el virus JC (JCPyV) ${ }^{2}$ en pacientes con un cuadro clínico conocido como leucoencefalopatía multifocal progresiva (LEMP). Ambos son considerados como potenciales virus oncogénicos $\mathrm{y}$, si bien su relación con tumores en humanos es controversial, se ha sugerido una asociación de BKPyV a tumores cerebrales, adenocarcinomas, cáncer de próstata y vejiga y de JCPyV a mesoteliomas, tumores cerebrales, osteosarcomas y linfomas ${ }^{3,4}$.

BKPyV y JCPyV pertenecen a la familia Polyomaviridae, género Polyomavirus. Son virus desnudos de $45 \mathrm{~nm}$ de diámetro, que poseen una cápside de simetría icosaédrica. Su genoma está compuesto por ADN de doble hebra circular, aproximadamente de 5.000 pares de bases, asociado a histonas ${ }^{5}$. Los genomas de BKPyV y de JCPyV comparten una identidad de $70-72 \% 5$. El genoma viral posee tres regiones que se transcriben bidireccionalmente y que tienen regulación temporal: región regulatoria no codificante, que contiene el origen de replicación y regiones regulatorias para activar la transcripción viral. Esta región presenta una alta tasa de recombinación genética, presentando alta variabilidad; región codificante tempra$n a$, que codifica para dos proteínas, una llamada antígeno tumoral grande, que corresponde a la principal proteína transformante requerida para el inicio de la replicación viral y otra llamada antígeno tumoral pequeño y región codificante tardía, que codifica para proteínas de cápside viral VP1, VP2, VP3 y una proteína llamada agnoproteína, que participa de la regulación viral ${ }^{6}$.

Desde el punto de vista epidemiológico, BKPyV y JCPyV se encuentran ampliamente diseminados entre la población mundial, observándose que a la edad de 10 años hasta $90 \%$ de la población seroconvierte para BKPyV y entre 50-60\% para JCPyV. Esto indica que la transmisión de estos agentes se establece a muy temprana edad y posiblemente en el contacto estrecho dentro del núcleo familiar ${ }^{3}$. Si bien el modo de adquisición aún es controversial, se ha postulado la transmisión respiratoria, orino-oral y fecal-oral como las principales rutas de contagio. 
La infección primaria de BKPyV ocurre en la infancia y generalmente es asintomática. La infección produce persistencia viral en células renales y del tracto urinario ${ }^{3}$. Varios estudios han mostrado material genético amplificado de BKPyV desde orina de individuos inmunocompetentes. Se ha determinado que BKPyV establece una infección persistente, usualmente con niveles indetectables de replicación ${ }^{7}$, pudiendo reactivarse por un estado de inmunosupresión. En pacientes inmunosuprimidos la excreción viral en orina puede relacionarse a cistitis hemorrágica y nefropatía asociada a polyomavirus ${ }^{8}$.

El virus JC está ampliamente distribuido en la población; sin embargo, no se tiene conocimiento de cómo se transmite entre las personas. El hallazgo de linfocitos y otros tipos celulares infectados con JCPyV en las amígdalas ha permitido proponer la vía respiratoria como sitio de entrada, a pesar de que no se le encuentra con frecuencia en saliva u orofaringe. Estudios realizados en Japón han demostrado transmisión intra y extra familiar. Los tejidos en los cuales se le encuentra principalmente en el individuo infectado son: epitelio del tracto renal y del tracto digestivo superior e inferior lo que permite presumir que esos serían sus principales sitios de latencia. $\mathrm{Su}$ amplia diseminación a otros tejidos hace suponer que utilizaría la vía hematógena, posiblemente a través de los linfocitos, dado el compromiso ocasional del bazo y linfonodos, y el alto porcentaje en que se encuentra su ADN en células mononucleares periféricas de pacientes con LEMP y con distintas patologías inmunosupresoras $^{9}$. Si bien en pacientes inmunocompetentes no se ha observado asociación de la infección por virus JC con una enfermedad, en inmunocomprometidos se le asocia a nivel del SNC con LEMP, síndrome inflamatorio de reconstitución inmune, neuropatía de células granulares, meningitis y meningoencefalitis, y en el sistema urogenital con nefropatía.

A pesar de que la infección primaria por BKPyV y JCPyV cursa de forma subclínica, se ha documentado que son capaces de persistir por un período de tiempo indefinido, dando paso eventualmente a reactivación, principalmente en inmunosuprimidos, en quienes provocan enfermedades graves y frecuentemente fatales ${ }^{3}$. En las últimas décadas ha existido un aumento de pacientes inmunosuprimidos $y$, en ese contexto, los polyomavirus se han comportado como patógenos emergentes en este tipo de pacientes.

En Chile no se han llevado a cabo estudios de prevalencia de polyomavirus BKPyV y JCPyV en pacientes infectados con VIH, por esa razón en este estudio nos propusimos identificar la presencia del genoma de estos virus en muestras de sangre provenientes de pacientes con infección por VIH y buscar la asociación con parámetros clínicos relacionados a la infección por VIH.

\section{Material y Método}

\section{Muestras}

Noventa y seis muestras de sangre de pacientes adultos con infección por VIH fueron obtenidas de la seroteca del Programa de Virología, ICBM, de la Facultad de Medicina de la Universidad de Chile. Correspondían a muestras obtenidas durante los años 2005 y 2006 para un proyecto de investigación virológico previamente diseñado y aprobado por el Comité de Ética respectivo, en el cual se reclutaron pacientes infectados por VIH adultos, que acudían a sus controles regulares al Servicio de Inmunología del Hospital San José y que accedieron a participar voluntariamente y mediante consentimiento informado escrito. Específicamente, las fracciones leucocitarias de dichas muestras fueron mantenidas a $-80^{\circ} \mathrm{C}$ hasta el presente análisis. Asimismo, 12 muestras de pacientes negativos para VIH fueron procesadas en paralelo.

Los datos socio-demográficos y clínicos relacionados a la infección por VIH que se tienen de estas muestras permiten total confidencialidad de los pacientes, ya que están registrados en una plantilla creada $a d-h o c$, la cual no admite identificar a los pacientes que participaron en el estudio original. El presente estudio fue aprobado por el Comité de Ética de la Institución.

\section{Detección de los genomas virales}

Para la extracción de ADN, las fracciones leucocitarias de las muestras de sangre fueron procesadas con un kit de purificación de ácido nucleico viral por columna (QIAgen ${ }^{\circledR}$ ) según las indicaciones del fabricante. El ADN purificado fue almacenado a $-20^{\circ} \mathrm{C}$ hasta su posterior uso en reacciones de polimerasa en cadena (RPC).

Cada extracción de ADN fue sometida inicialmente a un análisis para verificar la idoneidad como sustrato de detección. Para ello, se amplificó por RPC convencional un segmento correspondiente al gen de $\beta$-globina presente en el genoma celular ${ }^{10}$ y posterior detección en geles de agarosa al $1 \%$ en TBE.

La detección del genoma viral de BKPyV se realizó utilizando una modificación del protocolo de RPC en tiempo real descrito por Hoffman y cols. ${ }^{11}$, llamado V3T3. Los pares de partidores (V3 y T3) están diseñados para la amplificación de segmentos de genes virales que codifican para la proteína de cápside viral (VP1) y el antígeno tumoral mayor, respectivamente. Ambos blancos de amplificación fueron seleccionados por ser relativamente conservados y su amplificación se efectuó en tubos separados. Las condiciones de amplificación de BKPyV fueron: $50^{\circ} \mathrm{C}$ por dos minutos (descontaminación UDG) y $95^{\circ} \mathrm{C}$ por $10 \mathrm{~min}$, seguido de 45 ciclos de $95^{\circ} \mathrm{C}$ durante 20 seg y $60^{\circ} \mathrm{C}$ durante un minuto. Como control positivo se usó diluciones de un genoma de BKPyV clonado en plasmidio. Después del análisis en tiempo 
Tabla 1. Asociación entre características socio-demográficas y clínicas relacionadas a la infección por VIH de los pacientes y la presencia de BKV

\begin{tabular}{|c|c|c|c|c|c|}
\hline Características & & Pacientes & BKV Positivo & BKV Negativo & Valor $p^{a}$ \\
\hline Sexo & $\begin{array}{r}\mathrm{n} \text { total }^{\mathrm{b}} \\
\text { masculino } \\
(\%)\end{array}$ & $\begin{array}{c}n=83 \\
74 \\
(89,2)\end{array}$ & $\begin{array}{l}n=29 \\
28 \\
(96,6)\end{array}$ & $\begin{array}{c}n=54 \\
46 \\
(85,2)\end{array}$ & $0,112^{c}$ \\
\hline Edad (años) & $\begin{array}{r}\mathrm{n} \text { total }^{\mathrm{b}} \\
\text { mediana } \\
\text { rango }\end{array}$ & $\begin{array}{c}n=83 \\
37 \\
18-65\end{array}$ & $\begin{array}{c}n=29 \\
36 \\
18-65\end{array}$ & $\begin{array}{c}n=54 \\
38,5 \\
18-63\end{array}$ & $0,390^{c}$ \\
\hline Presencia de ITS & $\begin{array}{r}\mathrm{n} \text { total }^{\mathrm{b}} \\
\text { positivo } \\
(\%)\end{array}$ & $\begin{array}{l}n=83 \\
53 \\
(64,9)\end{array}$ & $\begin{array}{c}n=29 \\
21 \\
(75,0)\end{array}$ & $\begin{array}{l}n=54 \\
32 \\
(61,4)\end{array}$ & $0,234^{c}$ \\
\hline $\begin{array}{l}\text { Tiempo de diagnóstico } \\
\text { de VIH (meses) }\end{array}$ & $\begin{array}{r}\mathrm{n} \text { total }^{\mathrm{b}} \\
\text { mediana } \\
\text { rango }\end{array}$ & $\begin{array}{c}n=73 \\
72 \\
0-420\end{array}$ & $\begin{array}{c}n=25 \\
60 \\
1-204\end{array}$ & $\begin{array}{c}n=48 \\
78 \\
0-420\end{array}$ & $0,408^{c}$ \\
\hline $\begin{array}{l}\text { Recuento de CD4 } \\
\left(\mathrm{LT}_{\mathrm{CD} 4} / \mu \mathrm{L}\right)\end{array}$ & $\begin{array}{r}\mathrm{n} \text { total }^{\mathrm{b}} \\
\text { mediana } \\
\text { rango }\end{array}$ & $\begin{array}{c}n=78 \\
366 \\
3-3.510\end{array}$ & $\begin{array}{l}n=29 \\
312 \\
3-3.510\end{array}$ & $\begin{array}{c}n=49 \\
411 \\
7-1.236\end{array}$ & $0,115^{c}$ \\
\hline $\begin{array}{l}\text { Carga viral VIH } \\
\text { Detectable }\end{array}$ & $\begin{array}{l}\mathrm{n} \text { total }^{\mathrm{b}} \\
\text { positivo } \\
(\%)\end{array}$ & $\begin{array}{c}n=78 \\
33 \\
42,3\end{array}$ & $\begin{array}{c}n=29 \\
8 \\
27,6\end{array}$ & $\begin{array}{l}n=49 \\
25 \\
51,0\end{array}$ & 0,043 \\
\hline $\begin{array}{l}\text { Carga viral VIH } \\
\text { (copias } / \mathrm{mL})\end{array}$ & $\begin{array}{r}\mathrm{n} \text { total }^{\mathrm{b}} \\
\text { mediana } \\
\text { rango }\end{array}$ & $\begin{array}{c}n=78 \\
0^{d} \\
0^{d}-1.200 .000\end{array}$ & $\begin{array}{c}n=29 \\
0^{d} \\
0^{d}-1.200 .000\end{array}$ & $\begin{array}{c}n=49 \\
180 \\
0^{d}-380.000\end{array}$ & $0,067^{c}$ \\
\hline
\end{tabular}

aMann-Whitney para: Edad, Tiempo de diagnóstico, Recuento de CD4 y Carga viral. Pearson para: Sexo, Presencia de infecciones de transmisión sexual (ITS) y Carga viral detectable. bNo están disponibles todos los datos de todos los pacientes, por eso se especifica el $n$ analizado de cada característica. 'No significativo. ${ }^{d}$ No detectable.

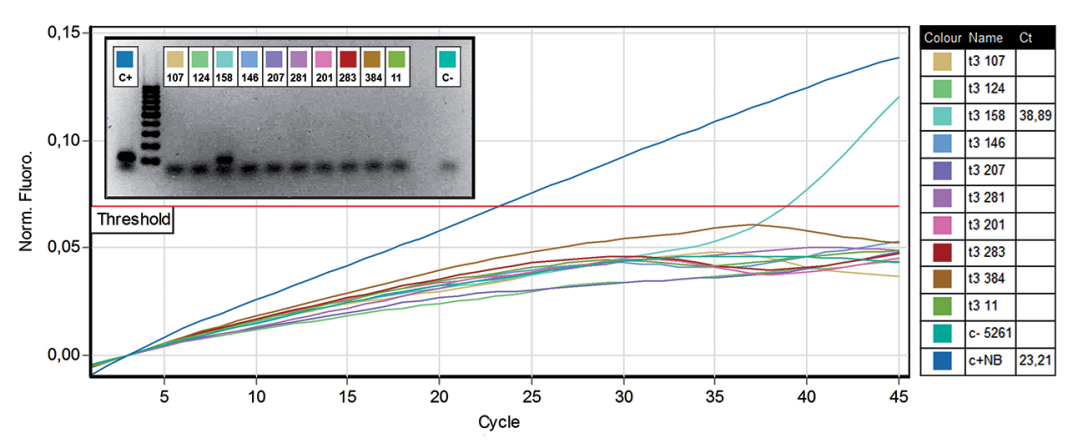

Figura 1. Detección del amplicón T3 de BKPyV en 10 muestras. Tanto por sonda como por electroforesis (gráfico inserto), la muestra 158 evidencia amplificación. Las otras muestras son todas negativas para este segmento. C+: control positivo; C- Control negativo.

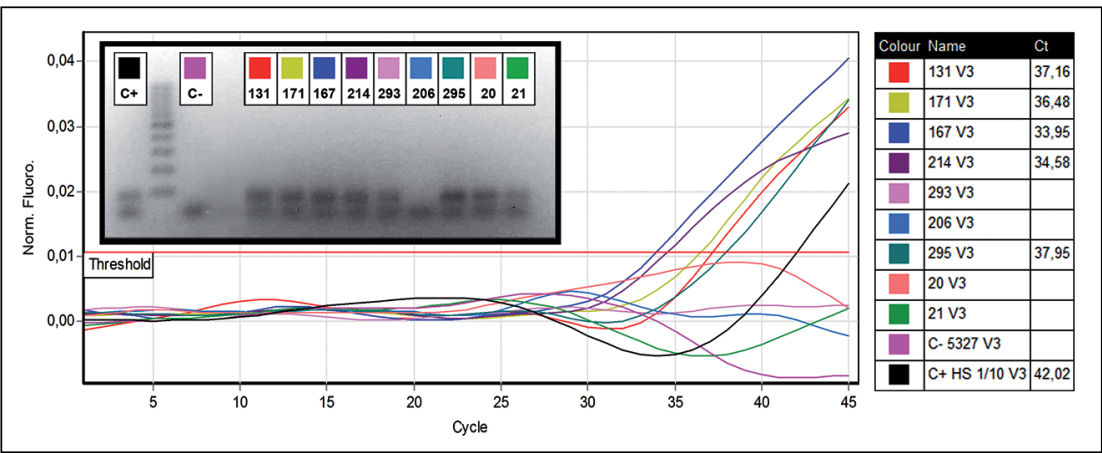

Figura 2. Detección del amplicón V3 de BKPyV en 10 muestras. Las muestras 131, 171, 167, 214 y 295 son detectadas tanto por sonda como por electroforesis (inserto), mientras que las muestras 293, 20 y 21 sólo muestran amplificación por electroforesis y no por sonda. real, los ensayos de RPC de BKPyV fueron sometidos a electroforesis en gel de agarosa al $2 \%$ a $100 \mathrm{~V}$ por $45 \mathrm{~min}$. Luego de 10 min en bromuro de etidio, se observaron las bandas de material genético amplificado (97 pb para T3 y 79 pb para V3). De esta manera se cuenta con cuatro criterios de detección del genoma de $\mathrm{BKPyV}$.

Para la detección del genoma de JCPyV se utilizó el protocolo descrito por Pal y cols., específicamente con el par de partidores JE3 dirigidos al gen codificante del antígeno mayor ${ }^{12}$. Las condiciones de amplificación fueron: descontaminación UDG $50^{\circ} \mathrm{C}$ por dos minutos, denaturación inicial $94{ }^{\circ} \mathrm{C}$ por 10 min seguido de 50 ciclos de denaturación $94{ }^{\circ} \mathrm{C}$ por $10 \mathrm{seg}$, alineamiento de $50{ }^{\circ} \mathrm{C}$ por $30 \mathrm{seg}$ y extensión $60{ }^{\circ} \mathrm{C}$ por $30 \mathrm{seg}$. El control positivo usado fueron diluciones de un plasmidio (pGEM-T-Easy) con el mismo amplicón insertado en el sitio de clonamiento.

Las sondas de hidrólisis TaqMan para ambos ensayos fueron marcadas con FAM (6-carboxifluoresceína) en el extremo 5' y con un quencher BBQ en el extremo 3'. Todas las operaciones de RPC en tiempo real se efectuaron en el termociclador RotorGene, CORBETT, modelo RG6000. La adquisición de datos se hizo a 530 nanómetros en la etapa de $60^{\circ} \mathrm{C}$ de cada ciclo. El punto de cruce (CP) se determinó utilizando el software incorporado al equipo.

\section{Análisis estadístico}

Para contrastar la presencia de BKPyV y JCPyV en las muestras de sangre con los datos socio-demográficos registrados anteriormente de éstas, se utilizó la prueba de $\chi^{2}$ de Pearson para variables categóricas y la prueba de Mann-Whitney para variables continuas en el programa SPSS Statistics 18.0. Se consideró significativo un valor de $\mathrm{p}<0,05$.

\section{Resultados}

Todas las muestras de pacientes sin infección VIH resultaron positivas para $\beta$-globina y negativas para BKPyV y JCPyV.

De las 96 muestras de pacientes con VIH incorporadas al estudio, siete de ellas no mostraron amplificación del gen de $\beta$-globina, por lo que la búsqueda de genomas virales sólo se continuó en las 89 positivas.

Treinta muestras (33,7\%) fueron positivas para BKPyV por al menos uno de los cuatro criterios utilizados. De ellas: tres muestras resultaron positivas por todos los criterios; dos muestras resultaron positivas sólo para T3 (sonda y electroforesis, ejemplo en la Figura 1); 15 muestras sólo para V3 (sonda y electroforesis) y 10 muestras fueron positivas solamente por electroforesis en gel (todas del amplicón V3), como se observa en la Figura 2, lo que sugiere un cierto grado de variabilidad en la región blanco de la sonda. 
Todas las muestras resultaron negativas en lo referido a la detección del genoma de JCPyV, a pesar de la consistencia del control positivo.

Las características socio-demográficas y clínicas de los pacientes se describen en la Tabla 1. Sólo en seis pacientes no se registraron datos. Al realizar el análisis estadístico de las variables con los resultados obtenidos para BKPyV, se observó una relación estadísticamente significativa entre la ausencia de carga viral detectable de VIH y la presencia de BKPyV ( $p=0,043$, por test de Pearson).

\section{Discusión}

El hallazgo más significativo de este estudio es haber determinado la presencia de genoma viral de virus BK en los leucocitos de $33 \%$ de las muestras de sangre de pacientes infectados con VIH. Estudios previos en pacientes sanos habían mostrado prevalencias muy diversas que oscilaron entre $7 \%$ y $92 \%$ en muestras de leucocitos ${ }^{13}$. En pacientes inmunocomprometidos también se ha determinado porcentajes diversos entre $4,7 \%$ en países nórdicos y $25 \%$ en Italia con técnicas más recientes ${ }^{14,15}$; no puede excluirse la posibilidad de que la mejora en la sensibilidad de las técnicas de diagnóstico haya sido un factor relevante en la obtención de datos tan disímiles. Por otro lado, la mayor parte de estudios se ha focalizado en la búsqueda de genoma viral en muestras de orina, donde presuntamente se estaría detectando partículas virales; la búsqueda en leucocitos periféricos, tal como en este trabajo, está más focalizado en la búsqueda de genomas persistentes en células, donde no necesariamente está habiendo replicación activa, pero que podrían llevar a una reactivación posterior.

Los datos aquí mostrados sugieren una correlación inversa entre la detección de virus BK en leucocitos y la presencia de concentraciones detectables de VIH en suero (Tabla 1). Dado que el principal tropismo del virus VIH son sub-poblaciones de linfocitos, es muy tentadora la hipótesis de que exista alguna suerte de bloqueo o competencia entre ambos virus haciendo que sólo uno de ellos pueda infectar un conjunto de células. En estudios previos y utilizando muestras de orina (replicación activa del virus) se reportaron datos contradictorios al respecto: tanto en Inglaterra como en Estados Unidos de América se encontró que más pacientes con recuentos de CD4+ bajo $200 / \mathrm{mm}^{3}$ excretaron ADN viral en la orina ${ }^{16,17}$, mientras que en Brasil no se observaron diferencias al respecto ${ }^{18}$.

La nula presencia de virus JC en leucocitos no es del todo sorprendente, considerando que estudios previos, tanto en pacientes con VIH como en pacientes normales ya se habían mostrado prevalencias bajas o nulas en este tipo de muestras ${ }^{14,19-21}$ y la excepción hasta ahora han sido pacientes que han sido diagnosticados con LEMP $\mathrm{u}$ otras enfermedades neurológicas ${ }^{22,23}$. Nuestro grupo de pacientes estudiados no tenían estos cuadros clínicos.
Desde que se instalaron las técnicas de amplificación de ácidos nucleicos, ha sido muy difícil consensuar un protocolo único para el diagnóstico tanto de BKPyV como de JCPyV. Sólo el año 2014 fue posible encontrar en Medline reportes con cinco protocolos distintos para la detección de estos polyomavirus en muestras clínicas ${ }^{24-28}$. Esto se debe a que existe una importante diversidad de secuencias y no se ha establecido regiones conservadas absolutas. Por esa razón, para la búsqueda de BKPyV utilizamos cuatro criterios distintos: dos blancos de amplificación (genes VP1 y antígeno T) y cada uno de ellos con o sin sonda específica (qPCR y electroforesis). Los resultados confirmaron la necesidad de utilizar más de un criterio, puesto que sólo tres de las 30 muestras positivas lo fueron por todos los criterios, mientras que dos lo fueron sólo con los partidores de antígeno $\mathrm{T}$ y 25 sólo con los partidores de VP1, incluidas 10 muestras que fueron positivas sólo por amplificación pero no detectables con la sonda utilizada. Esta dificultad aumenta la posibilidad de que los estudios previos hayan subestimando la prevalencia real de polyomavirus en humanos. Futuros estudios realizados por medio de secuenciamiento masivo de ácidos nucleicos permitirán establecer con mayor precisión la riqueza genética de las poblaciones de estos virus.

Los cuadros clínicos atribuidos a BKPyV y JCPyV son más bien infrecuentes respecto al total de la población infectada. Sin embargo, en pacientes inmunosuprimidos el riesgo tanto de cistitis hemorrágicas y nefropatías causadas por BKPyV como de LEMP debida a JCPyV es considerablemente mayor, lo que refuerza la tesis de que dichos pacientes padecen una infección latente que se reactiva ante la inmunosupresión ${ }^{3}$. Considerando lo anterior es que en este estudio se buscó la presencia de genomas virales en leucocitos periféricos, como posible sitio de persistencia, de pacientes infectados con VIH, heterogéneos en su estado inmunológico. El alto porcentaje de pacientes positivos para BKPyV que aquí se reporta permite sugerir que dichos pacientes podrían tener mayores probabilidades de complicaciones en el sistema urinario y por lo tanto requerirían controles urológicos y nefrológicos periódicos. Futuros estudios que analicen la presencia de virus en plasma y otras secreciones podrían además facilitar una hipótesis respecto al modo y frecuencia de transmisión del virus, particularmente entre pacientes infectados con VIH.

\section{Resumen}

Introducción: Polyomavirus BK (BKPyV) y JC (JCPyV) son patógenos persistentes con capacidad de reactivación en inmunocomprometidos, afectando principalmente el sistema urinario y el sistema nervioso central, respectivamente. No existen estudios chilenos en población infectada por VIH. Objetivo: Detectar la presencia 
de BKPyV y JCPyV en muestras de sangre de pacientes adultos, chilenos, con infección por VIH y correlacionar los resultados con variables clínicas. Materiales y Métodos: Analizamos 96 muestras de extractos leucocitarios de pacientes del área norte de Santiago. El genoma viral se detectó mediante RPC en tiempo real. Para el análisis estadístico se utilizó las pruebas chi-cuadrado de Pearson y Mann-Whitney, considerando significativo un valor de $\mathrm{p}<0,05$. Resultados: $33 \%$ de las muestras resultaron positivas para BKPyV y se encontró una correlación significativa entre la presencia de genoma de BKPyV y la ausencia de carga viral de VIH. Se evidenció la necesidad de considerar más de un blanco de amplificación del genoma de BKPyV. Todas las muestras fueron negativas para JCPyV. Discusión: La prevalencia de BKPyV en pacientes chilenos con infección por VIH es superior a la informada en la mayoría de los reportes internacionales. Se requiere estudios que evalúen la interacción entre ambos virus. Estos pacientes deberían ser sometidos a controles urológicos y nefrológicos periódicos.

\section{Referencias bibliográficas}

1.- Gardner S D, Field A M, Coleman D V, Hulme B. New human papovavirus (B.K.) isolated from urine after renal transplantation. Lancet 1971; 1: 1253-7.

2.- Padgett B L, Walker D L, ZuRhein G M, Eckroade R J, Dessel B H. Cultivation of papova-like virus from human brain with progressive multifocal leucoencephalopathy. Lancet 1971; 1: 1257-60.

3.- Jiang M, Abend J R, Johnson S F, Imperiale M $\mathrm{J}$. The role of polyomaviruses in human disease. Virology 2009. p. 266-73

4.- Dalianis T, Hirsch H H. Human polyomaviruses in disease and cancer. Virology 2013. p. 63-72.

5.- Luo C, Bueno M, Kant J, Randhawa P. Biologic diversity of polyomavirus BK genomic sequences: Implications for molecular diagnostic laboratories. J Med Virol 2008; 80: 1850-7.

6.- Moens U, Johannessen M. Human polyomaviruses and cancer: expanding repertoire. J Dtsch Dermatol Ges 2008; 6: 704 8.

7.- White M K, Gordon J, Khalili K. The rapidly expanding family of human polyomaviruses: recent developments in understanding their life cycle and role in human pathology. PLoS Pathogens 2013: 9 (3): e1003206.

8.- Costa C, Bergallo M, Sidoti F, Astegiano S, Terlizzi M E, Mazzucco G, et al. Polyomaviruses BK- and JC-DNA quantitation in kidney allograft biopsies. J Clin Virol 2009; 44: 20-3.

9.- Khalili K, Gordon J, White M K. The polyomavirus, JCV, and its involvement in human disease. Adv Exp Med Biol 2006; 577 : 274-87.

10.- Saiki R K, Scharf S, Faloona F, Mullis K B, Horn G T, Erlich H A, et al. Enzymatic amplification of beta-globin genomic sequences and restriction site analysis for diagnosis of sickle cell anemia. Science (80- ). 1985; 230 (4732): 1350-4.

11.- Hoffman N G, Cook L, Atienza E E, Limaye A $\mathrm{P}$, Jerome K R. Marked variability of BK virus load measurement using quantitative real-time PCR among commonly used assays. J Clin
Microbiol 2008; 46 (8): 2671-80.

12.- Pal A, Sirota L, Maudru T, Peden K, Lewis A M. Real-time, quantitative PCR assays for the detection of virus-specific DNA in samples with mixed populations of polyomaviruses. J Virol Methods 2006; 135 (1): 32-42.

13.- Knowles W A. Discovery and epidemiology of the human polyomaviruses BK virus (BKV) and JC virus (JCV). Adv Exp Med Biol 2006; 577 : 19-45.

14.- Sundsfjord A, Flaegstad T, Flø R, Spein A R, Pedersen M, Permin H, et al. BK and JC viruses in human immunodeficiency virus type 1-infected persons: prevalence, excretion, viremia, and viral regulatory regions. J Infect Dis 1994; 169 (3): 485-90.

15.- Delbue S, Tremolada S, Elia F, Carloni C, Amico S, Tavazzi E, et al. Lymphotropic polyomavirus is detected in peripheral blood from immunocompromised and healthy subjects. J Clin Virol 2010; 47: 156-60.

16.- Knowles W A, Pillay D, Johnson M A, Hand J F, Brown D W G. Prevalence of long-term BK and JC excretion in HIV-infected adults and lack of correlation with serological markers. J Med Virol 1999; 59 (4): 474-9.

17.- Markowitz R B, Thompson H C, Mueller J F, Cohen J A, Dynan W S. Incidence of $\mathrm{BK}$ virus and $\mathrm{JC}$ virus viruria in human immunodeficiency virus -infected anduninfected subjects. J Infect Dis 1993; 167 (1): 13-20.

18.- Nali L H da S, Centrone C de C, Urbano P R P, Penalva-de-Oliveira A C, Vidal J E, Miranda E P, et al. High prevalence of the simultaneous excretion of polyomaviruses $\mathrm{JC}$ and $\mathrm{BK}$ in the urine of HIV-infected patients without neurological symptoms in São Paulo, Brazil. Rev Inst Med Trop Sao Paulo 2012; 54 (4): 201-5.

19.- Comar M, Zanotta N, Bovenzi M, Campello C. JCV/BKV and SV40 viral load in lymphoid tissues of young immunocompetent children from an area of north-east Italy. J Med Virol 2010; 82 (7): 1236-40.

20.- Bialasiewicz S, Whiley D M, Lambert S B, Nissen M D, Sloots T P. Detection of BK, JC, WU, or KI polyomaviruses in faecal, urine, blood, cerebrospinal fluid and respiratory samples. J Clin Virol 2009; 45 (3): 249-54.

21.- Domínguez-Mozo M I, García-Montojo M, De Las Heras V, García-Martínez A, Arias-Leal A M, Casanova I, et al. Anti-JCV antibodies detection and JCV DNA levels in PBMC, serum and urine in a cohort of Spanish Multiple Sclerosis patients treated with natalizumab. J Neuroimmune Pharmacol 2013; 8 (5): 1277-86.

22.- Koralnik I J, Boden D, Mai V X, Lord C I, Letvin N L. JC virus DNA load in patients with and without progressive multifocal leukoencephalopathy. Neurology 1999; 52 (2): 253-60.

23.- Bellizzi A, Anzivino E, Rodio D M, Cioccolo S, Scrivo R, Morreale M, et al. Human polyomavirus JC monitoring and noncoding control region analysis in dynamic cohorts of individuals affected by immunemediated diseases under treatment with biologics: an observational study. Virol J 2013; 10: 298 .

24.- Alosaimi B, Hampson L, He X, Maranga I O, Oliver A W, Hampson I N. Increased prevalence of JC polyomavirus in cervical carcinomas from women infected with HIV. J Med Virol 2014; 86 (4): 672-7.

25.- Chattaraj S, Bhattacharjee S. Molecular analysis of JC polyomavirus genotypes circulating among tribal populations of North-Eastern West Bengal, India. Pol J Microbiol 2014; 63 (2): 191-201.

26.- Funahashi Y, Kato M, Fujita T, Takai S, Kimura Y, Gotoh M. Prevalence of polyomavirus positivity in urine after renal transplantation. Transpl Proc 2014; 46 (2): 564-6.

27.- Hasegawa M, Ito T, Saigo K, Akutsu N, Maruyama M, Otsuki K, et al. Association of DNA amplification with progress of BK polyomavirus infection and nephropathy in renal transplant recipients. Transpl Proc 2014; 46 (2): 556-9.

28.- Sadeghi F, Salehi-Vaziri M, Ghodsi S M, Alizadeh A, Bokharaei-Salim F, Saroukalaei S $\mathrm{T}$, et al. Prevalence of JC polyomavirus large T antigen sequences among Iranian patients with central nervous system tumors. Arch Virol 2015; 160 (1): 61-8. 